\title{
One-way electromagnetic Tamm states in magnetophotonic structures
}

\author{
Alexander B. Khanikaev, ${ }^{1, a)}$ Alexander V. Baryshev, ${ }^{2,3}$ Mitsuteru Inoue, $^{2}$ and \\ Yuri S. Kivshar ${ }^{4}$ \\ ${ }^{1} M Q$ Photonics Research Centre, Department of Physics and Engineering, Macquarie University, \\ Sydney NSW 2109, Australia \\ ${ }^{2}$ Toyohashi University of Technology, Toyohashi, Aichi 441-8580, Japan \\ ${ }^{3}$ Ioffe Physico-Technical Institute, St. Petersburg 194021, Russia \\ ${ }^{4}$ Nonlinear Physics Center, Research School of Physics and Engineering, Australian National University, \\ Canberra, Australian Capital Territory 0200, Australia
}

(Received 30 May 2009; accepted 10 June 2009; published online 6 July 2009)

\begin{abstract}
We study surface Tamm states in magnetophotonic structures magnetized in the Cotton-Mouton (Voigt) geometry. We demonstrate that the periodicity violation due to the structure truncation together with the violation of the time reversal symmetry due to the presence of magneto-optical materials gives rise to nonreciprocality of the surface modes. Dispersion of forward and backward modes splits and becomes magnetization dependent. This results in the magnetization-induced transitions between bulk and surface modes and unidirectional propagation of surface waves.
\end{abstract} (C) 2009 American Institute of Physics. [DOI: 10.1063/1.3167356]

Surface electromagnetic waves, which exist at the interfaces separating different media, are proven to be important in many applications ranging from integrated optical circuits to biosensors. One of the most known types of such waves are surface plasmon-polaritons (SPPs), which exist at interfaces separating metals and dielectrics. ${ }^{1}$ The other types of commonly known surface waves are optical Tamm states (OTS), which exist at the surface of photonic crystals (PCs). ${ }^{2-4}$ Although these two types of surface waves have different natures, their properties are quite similar. In particular, dispersion of these excitations can be engineered by an appropriate structuring of metal surfaces ${ }^{5-8}$ or design of $\mathrm{PCs},{ }^{2-4,9}$ respectively. A great advantage of the photonic surface modes such as OTSs in comparison with SPPs is their low attenuation. Thus, OTSs represent an excellent alternative for a variety of optical elements with a functionality relying on the surface waves. SPPs have been known for many years to exhibit nonreciprocal behavior in the presence of an external magnetic field. ${ }^{1}$ However, this nonreciprocity is usually quite marginal, and/or it requires very large magnetic fields. Nevertheless, quite interesting phenomena were recently predicted for systems supporting these excitations. ${ }^{10}$

The present work aims to introduce a concept of nonreciprocity for OTSs that exist at interface of magnetophotonic crystals (MPCs) (Ref. 11) and dielectrics, and explore the possibility to tailor their dispersion by magnetization and engineer the nonreciprocal response by an appropriate design of the structure. There are reasons to expect that, in contrast to SPPs, nonreciprocity of OTSs in MPCs reveals itself in relatively weak magnetic fields. ${ }^{11-17}$

In the following, we limit our consideration to onedimensional MPCs. We consider two-component MPCs with an elementary cell consisting of one isotropic and one magneto-optical active layer homogeneously magnetized in the Cotton-Mouton (Voigt) geometry (see the inset in Fig. 1). We obtain in an exact analytical form dispersion law of the OTSs localized at the boundary of such MPC. We demonstrate that dispersion of these OTSs is nonreciprocal. It

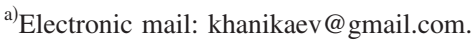

also depends on magnetization and therefore is tunable.

Nonreciprocity is a subtle property of magnetic materials. In addition to violation of the time reversal symmetry it also requires removal of the mirror reflection symmetry. ${ }^{15-17}$ The latter can be achieved by truncation of the periodic structure- it is the surface responsible for the existence of OTSs bound to the interface of PCs. Therefore, we can expect that even if Bloch modes supported by MPCs are reciprocal,${ }^{17}$ nonreciprocity will appear in semi-infinite or finite structures.

To find dispersion of OTSs we use the transfer matrix approach. ${ }^{2,17,18}$ First, we find the Bloch modes supported by the periodic structure. This step formally can be written in the form of the eigenvalue problem $\hat{T} \bar{H}_{y}=\exp \left(i K a_{0}\right) \bar{H}_{y}$, where $K$ is a Bloch number, $\bar{H}_{y}=\left(H_{y}^{+}, H_{y}^{-}\right)^{T}, a_{0}=a_{1}+a_{2}$ is the lattice constant with the layers forming an elementary cell $a_{1}$ and $a_{2}$, respectively, $\hat{T}=\hat{M}_{21} \hat{P}_{2} \hat{M}_{12} \hat{P}_{1}$ is the total transfer ma-

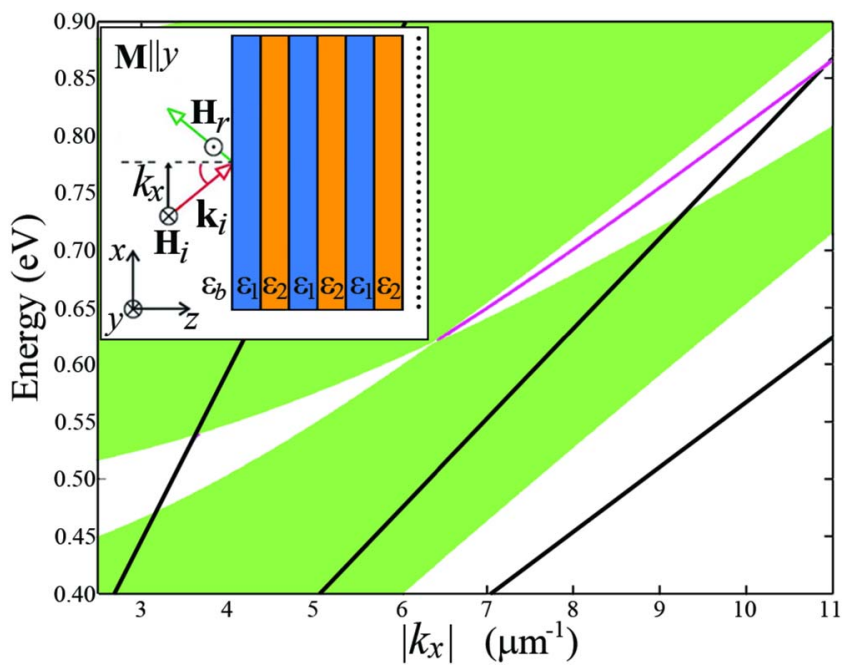

FIG. 1. (Color online) Dispersion of OTS in the nonmagnetized MPC (magenta line). Green and white regions correspond to bands and gaps of an infinite structure. Black lines show light lines in dielectrics of MPC and the background. Inset shows geometry of the problem. 
trix of an elementary cell, and $\hat{M}_{i j}$ is the interface matrix relating the magnetic field amplitudes of the forward $\left(H_{y}^{+}\right)$ and backward $\left(H_{y}^{-}\right)$waves at two opposite sides of a particular interface between the layers $i$ and $j$ within the unit cell $\left[\bar{H}_{y}\right]_{j}=\hat{M}_{i j}\left[\bar{H}_{y}\right]_{i}$, and it has the form

$$
\hat{M}_{i j}=\frac{d_{j}}{2 \epsilon_{j} k_{z j}}\left(\begin{array}{cc}
F_{j}^{*}+F_{i} & F_{j}^{*}-F_{i}^{*} \\
F_{j}-F_{i} & F_{j}+F_{i}^{*}
\end{array}\right),
$$

where $\quad F_{m}=\left(\epsilon_{m} k_{z m}+i \Delta_{m} k_{x m}\right) / d_{m}$. Matrix $\quad \hat{P}_{m}$ $=\operatorname{diag}\left[\exp \left(i k_{z m} a_{m}\right), \exp \left(-i k_{z m} a_{m}\right)\right]$ accounts for the phase shift accumulated by the wave during propagation in $m$-th layer, $d_{m}=\epsilon_{m}^{2}-\Delta_{m}^{2}, k_{m}=n_{m} k_{0}$ is the modulus of the wave vector, $n_{m}=\sqrt{d_{m} / \epsilon_{m}}$ is the refractive index, and the permittivity tensor $\hat{\epsilon}_{m}$ satisfies $\epsilon_{m, x x}=\epsilon_{m, y y}=\epsilon_{m, z z}$ and $\epsilon_{m, x z}=-\epsilon_{m, z x}=i \Delta_{m}$. The solution of the Floquet-Bloch eigenvalue problem allows one to write down Bloch modes of infinite MPC in an explicit form. ${ }^{2,18}$

When the first part of the problem is solved, we make the modal matching, i.e., impose an additional boundary conditions for the interface between MPC and surrounding homogeneous dielectric background, which in general case can be magnetic, and characterized by the dielectric and magneto-optical parameters $\epsilon_{b}$ and $\Delta_{b}$, respectively. Here we limit our analysis to the case of semi-infinite MPC and therefore conduct modal matching at the interface between the terminating layer of the MPC and the background. Calculations give the following dispersion law for the OTSs:

$$
q=i k_{1 z} \frac{n_{b}^{2}}{n_{1}^{2}} \frac{\widetilde{T}_{12}+T_{11}-e^{i K a_{0}}}{\widetilde{T}_{12}-T_{11}+e^{i K a_{0}}}+k_{x}\left(\frac{\Delta_{1}}{\varepsilon_{1}} \frac{n_{b}^{2}}{n_{1}^{2}}-\frac{\Delta_{b}}{\varepsilon_{b}}\right),
$$

where $q$ is a wave decay rate in the background material, ${ }^{2,18}$ $\widetilde{T}_{12}=T_{12} \exp \left(-2 i k_{1 z} \sigma a_{1}\right)$, and parameter $\sigma$ determines truncation of the terminating layer of the structure (the dielectric 1 here) so that its thickness is equal to $\sigma a_{1}$.

The effect of magnetization on dispersion of OTSs can be separated into two different types of contribution. The contribution of the first type is explicitly reflected in an additional magnetization-dependent second term on the right hand side of Eq. (2), (this term is not present in the case of nonmagnetic PCs). ${ }^{2,18}$ Nonreciprocal contribution of this term is evident, because of its linear dependence on the wave vector component $k_{x}$. Note that this type of contribution incorporates nonreciprocity additively and thus does not benefit in any way from the periodicity of MPC and the existence of the photonic band structure. In fact, the mechanism of the nonreciprocity behind this contribution has the same origin as the nonreciprocity in a trivial planar dielectric waveguide or a metal surface for the case of SPPs. It comes exclusively from the requirement of the continuity of the tangential component of the electric field at the interface between materials of the background and the terminating layer of MPC. Its contribution vanishes if neither of these two materials is magnetic regardless of the presence of magnetic layers in the depth of MPC. This type of nonreciprocity is trivial and will not be considered here.

The second type of contribution to the nonreciprocity has a completely different origin and appears due to violation of the periodicity in truncated MPCs. It comes from the first term on the right hand side of Eq. (2) which is directly related to the periodicity of the MPC. This is because this term

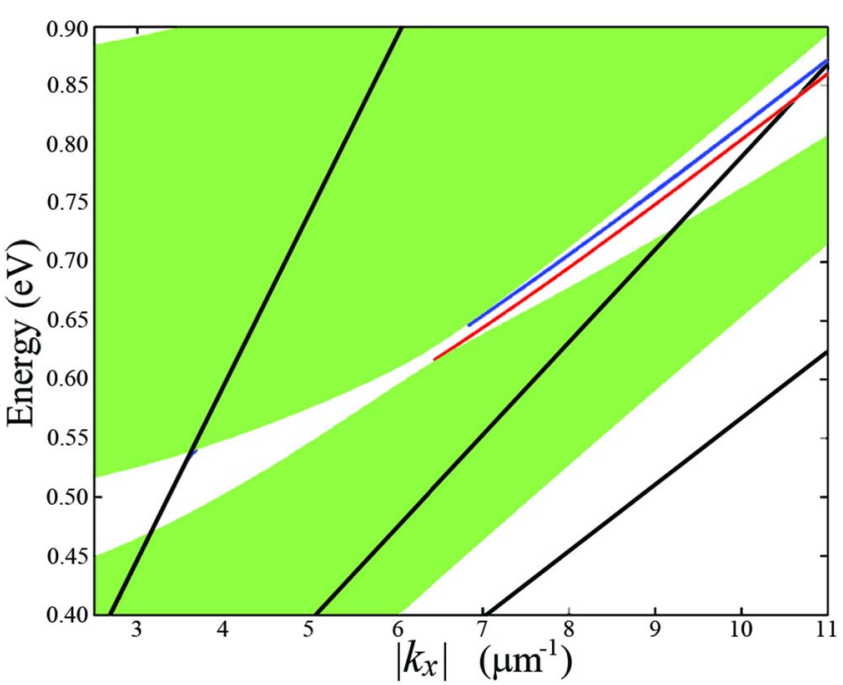

FIG. 2. (Color online) Same as in Fig. 1, the semi-infinite MPC is magnetized. Red and blue lines correspond to the forward and backward propagating OTS, respectively.

incorporates matrix elements $T_{11}$ and $T_{12}$ of the transfer matrix of the elementary cell and Bloch number $K$. Note that for infinite two-component MPCs dispersion is always reciprocal, i.e., $K\left(k_{x}\right)=K\left(-k_{x}\right) .{ }^{17}$ Thus, the nonreciprocity develops exclusively from the transfer matrix terms in Eq. (2).

To demonstrate that this type of nonreciprocity does really show up and surface modes possess a nonreciprocal dispersion, Eq. (2) was solved numerically for the case of $\mathrm{Si} /$ BIG $\left(\varepsilon_{1}=\varepsilon_{\mathrm{Si}}=12, \quad \varepsilon_{2}=\varepsilon_{\mathrm{BIG}}=6.25\right)$ semi-infinite MPC terminated by $\sigma=0.7$ fraction of the Si layer $\left(\Delta_{1}=\Delta_{\mathrm{Si}}=0\right)$ and embedded into the air background $\left(\varepsilon_{b}=\varepsilon_{\text {air }}=1\right.$ and $\Delta_{b}$ $=\Delta_{\text {air }}=0$ ). This configuration excludes contribution from the second term of Eq. (2) and allows us to concentrate only on the nonreciprocity associated with the periodicity violation. For the sake of illustration we put a nondiagonal element of the permittivity tensor $\Delta_{2}=\Delta_{\mathrm{BIG}}=0.3$, which is $\approx 5$ times larger than its real value in Ce:BIG at optical communication wavelength. ${ }^{16}$

To reveal the effect of magnetization on dispersion of OTSs, it is more instructive to compare cases of magnetized and nonmagnetized structures. Calculation result for the latter case is given in Fig. 1, which shows regions of photonic pass-bands (green regions) and stop-gaps (white regions) together with the dispersion of the OTS. As can be seen from Fig. 1, the nonmagnetized PC supports one surface mode within the first band gap region. As expected, dispersion of this mode satisfies the reciprocity condition $\omega\left(k_{x}\right)=\omega\left(-k_{x}\right)$. If, however, the structure is magnetized (Fig. 2), then dispersion of the OTS changes, and, what is important, becomes nonreciprocal $\omega\left(k_{x}\right) \neq \omega\left(-k_{x}\right)$. Figure 2 shows dispersion of the forward $\left(k_{x}>0\right)$ and backward $\left(k_{x}<0\right)$ OTSs by red and blue lines, respectively. It is clear that these waves have different dispersion and therefore are nonreciprocal. Thus, calculations showed that presence of the interface in semiinfinite MPC was a sufficient condition for appearance of the nonreciprocality in the dispersion of OTSs. Note that reversal of the magnetization direction has the same effect as reversal of the propagation direction $\left(k_{x} \rightarrow-k_{x}\right)$.

From the results presented above, it becomes clear that magnetization causes a spectral splitting in the dispersion of waves propagating in the opposite directions, namely, disper- 


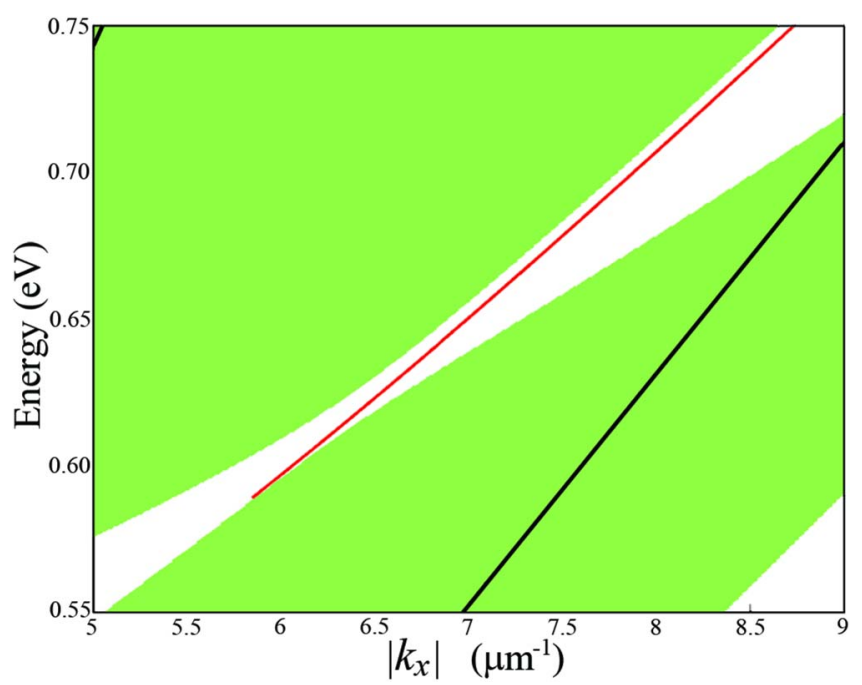

FIG. 3. (Color online) Dispersion of a unidirectional OTS (red line) for a truncated MPC. Solution for the opposite direction does not exist within the entire frequency range.

sion becomes redshifted or blueshifted as compared to nonmagnetized MPC. These spectral shifts give rise to one unique property of the nonreciprocal OTSs; they may become unidirectional. As can be seen in Fig. 2, the forward and backward modes have different cutoff frequencies. Thus, there is a frequency range near the cutoff where only the forward OTS can propagate, while the backward OTS is not supported by the same structure.

Now, when the effect of magnetization on the dispersion of OTSs is known, we can design a structure with a broadband unidirectionality. The main requirement for such a behavior is that the magnetization-induced splitting of the dispersion curves for the forward and backward waves should be sufficient to move the dispersion curves between the gap and band, thus implying a switching between surface and bulk states. In this case one of the modes would be decoupled from the surface whereas the other mode (propagating in the opposite direction) will remain a true surface mode.

To demonstrate the possibility of such transformation of OTSs into bulk modes we solved Eq. (2) for the structure with an appropriately truncated terminating layer. ${ }^{9}$ Figure 3 shows that magnetization-induced transition between surface waves and bulk waves in one direction can be achieved through an appropriate design of the structure $(\sigma=0.5)$. The forward/backward degenerate surface mode which exists near the band edge in the band gap of the nonmagnetized MPC splits into two modes in the magnetized structure. One of the modes, corresponding to the backward propagation, is blueshifted, and it moves inside the band, so that this mode is no longer a surface mode. In contrast, the other mode is shifted deeper into the gap, and it still represents a surface mode. Note that the opposite situation is also possible when magnetization induces a new surface mode by moving one wave into the gap.

In conclusion, we have predicted that surface waves supported by truncated magnetophotonic structures may exhibit nonreciprocal behavior. We have demonstrated that their nonreciprocity may give rise to extraordinary properties of OTSs making them unidirectional as well as may lead to magnetization-induced switching between regimes of surface and bulk modes.

We thank the Australian Research Council for a financial support and S. Fan for useful discussions. This work was supported in part by the Super Optical Information Memory Project from the Ministry of Education, Culture, Sports, Science and Technology of Japan (MEXT), and Grant-in-Aid for Scientific Research (S) Grant No. 17106004 from Japan Society for the Promotion of Science (JSPS).

${ }^{1}$ Surface Polaritons, edited by V. M. Agranovich and D. L. Mills (NorthHolland, Amsterdam, 1982); H. Raether, Surface Plasmons (Springer, Berlin, 1988).

${ }^{2}$ P. Yeh, A. Yariv, and C. Hong, J. Opt. Soc. Am. 67, 423 (1977); P. Yeh, A. Yariv, and A. Y. Cho, Appl. Phys. Lett. 32, 104 (1978).

${ }^{3}$ R. D. Meade, K. D. Brommer, A. M. Rappe, and J. D. Joannopoulos, Phys. Rev. B 44, 10961 (1991).

${ }^{4}$ A. Kavokin, I. Shelykh, and G. Malpuech, Appl. Phys. Lett. 87, 261105 (2005); Phys. Rev. B 72, 233102 (2005).

${ }^{5}$ I. V. Novikov and A. A. Maradudin, Phys. Rev. B 66, 035403 (2002).

${ }^{6}$ J. B. Pendry, L. Martín-Moreno, and F. J. Garcia-Vidal, Science 305, 847 (2004).

${ }^{7}$ D. F. P. Pile and D. K. Gramotnev, Opt. Lett. 29, 1069 (2004); 30, 1186 (2005).

${ }^{8}$ S. I. Bozhevolnyi, V. S. Volkov, E. Devaux, and T. W. Ebbesen, Phys. Rev. Lett. 95, 046802 (2005).

${ }^{9}$ C. Vandenbem, Opt. Lett. 33, 2260 (2008).

${ }^{10}$ Z. Yu, G. Veronis, Z. Wang, and S. Fan, Phys. Rev. Lett. 100, 023902 (2008)

${ }^{11}$ M. Inoue, R. Fujikawa, A. Baryshev, A. Khanikaev, P. B. Lim, H. Uchida O. Aktsipetrov, A. Fedyanin, T. Murzina, and A. Granovsky, J. Phys. D 39, R151 (2006).

${ }^{12}$ A. B. Khanikaev, A. B. Baryshev, P. B. Lim, H. Uchida, M. Inoue, A. G. Zhdanov, A. A. Fedyanin, A. I. Maydykovskiy, and O. A. Aktsipetrov, Phys. Rev. B 78, 193102 (2008).

${ }^{13}$ A. P. Vinogradov, A. V. Dorofeenko, S. G. Erokhin, M. Inoue, A. A. Lisyansky, A. M. Merzlikin, and A. B. Granovsky, Phys. Rev. B 74, 045128 (2006).

${ }^{14}$ T. Goto, A. V. Dorofeenko, A. M. Merzlikin, A. V. Baryshev, A. P. Vinogradov, M. Inoue, A. A. Lisyansky, and A. B. Granovsky, Phys. Rev. Lett. 101, 113902 (2008); T. Goto, A. V. Baryshev, M. Inoue, A. V. Dorofeenko, A. M. Merzlikin, A. P. Vinogradov, A. A. Lisyansky, and A. B. Granovsky, Phys. Rev. B 79, 125103 (2009).

${ }^{15}$ A. Figotin and I. Vitebskiy, Phys. Rev. E 63, 066609 (2001).

${ }^{16}$ Z. Yu, Z. Wang, and S. Fan, Appl. Phys. Lett. 90, 121133 (2007).

${ }^{17}$ A. B. Khanikaev and M. J. Steel, Opt. Express 17, 5265 (2009).

${ }^{18}$ M. Liscidini and J. E. Sipe, J. Opt. Soc. Am. B 26, 279 (2009). 